\title{
The Magpie Trial follow up study: outcome after discharge from hospital for women and children recruited to a trial comparing magnesium sulphate with placebo for pre-eclampsia [ISRCTN8693876I]
}

\author{
The Magpie Trial Follow Up Study Management Group* for The Magpie Trial \\ Follow Up Study Collaborative Group
}

Address: Magpie Trial Co-ordinating Centre, Institute of Health Sciences, Old Road, Headington, Oxford OX3 7LF, UK

Email: Magpie Trial Follow Up Study Management Group* - lelia.duley@ndm.ox.ac.uk; the Magpie Trial Follow Up Study Collaborative Group -

* Corresponding author

Published: 08 March 2004

BMC Pregnancy and Childbirth 2004, 4:5

This article is available from: http://www.biomedcentral.com/l47/-2393/4/5

(c) 2004 Magpie Trial Follow Up Study Management Group and Magpie Trial Follow Up Study Collaborative Group; licensee BioMed Central Ltd. This is an Open Access article: verbatim copying and redistribution of this article are permitted in all media for any purpose, provided this notice is preserved along with the article's original URL.

\begin{abstract}
Background: The Magpie Trial compared magnesium sulphate with placebo for women with preeclampsia. 10,14I women were recruited, 8804 before delivery. Overall, 9024 children were included in the analysis of outcome at discharge from hospital. Magnesium sulphate more than halved the risk of eclampsia, and probably reduced the risk of maternal death. There did not appear to be any substantive harmful effects on the baby, in the short term. It is now important to assess whether these benefits persist, and to provide adequate reassurance about longer term safety.

The main objective of the Magpie Trial Follow Up Study is to assess whether in utero exposure to magnesium sulphate has a clinically important effect on the child's chance of surviving without major neurosensory disability. Other objectives are to assess long term outcome for the mother, and to develop and assess appropriate strategies for following up large numbers of children in perinatal trials.
\end{abstract}

Study design: Follow up is only feasible in selected centres. We therefore anticipate contacting 2800-3350 families, for 2435-2915 of whom the woman was randomised before delivery. A further $280-335$ children would have been eligible for follow up if they had survived. The total sample size for the children is therefore 3080-3685, 2680-3210 of whom will have been born to women randomised before delivery.

Families eligible for the follow up will be contacted, and surviving children screened using the Ages and Stages Questionnaires. Children who screen positive, and a sample of those who screen negative, will whenever possible have a paediatric and neurodevelopmental assessment. When women are contacted to ask how their child is, they will also be asked about their own health. The primary outcome is a composite measure of death or neurosensory disability for the child at 18 months.

Discussion: The Follow Up Study began in 2002, and now involves collaborators in 19 countries. Data collection will close at the end of 2003. 


\section{Background}

Pre-eclampsia, a multisystem disorder of pregnancy usually associated with raised blood pressure and proteinuria, complicates $2-8 \%$ of pregnancies [1]. Although outcome is often good, pre-eclampsia is a major cause of morbidity and mortality for women and children $[2,3]$. It accounts for $10 \%$ of maternal deaths, and is also associated with increased perinatal mortality [4,5]. Eclampsia, the occurrence of seizures superimposed on pre-eclampsia, is rare. It affects 1 in 100 [6] to 1 in 2000 [7] deliveries, but is associated with a considerable increase in morbidity and mortality [7].

Magnesium sulphate is the anticonvulsant of choice for treating eclampsia [8-10]. The Magpie Trial has demonstrated it is also effective for preventing eclampsia [11,12]. Overall, this study recruited 10,141 women with preeclampsia. There was more than a halving in the risk of eclampsia (relative risk (RR) 0.42, 95\% confidence intervals (CI) 0.29 to 0.60 ) associated with the use of magnesium sulphate rather than placebo. The risk of maternal death was also reduced, although this did not reach statistical significance (RR $0.55,95 \%$ CI 0.26 to 1.14 ) [12]. There do not appear to be substantive harmful effects for the mother or baby, in the short term. In particular, there is reasonable reassurance that there was no clinically important effect on the risk of the baby dying before discharge from hospital or being in a special care nursery for more than 7 days (RR 1.02, 95\% CI 0.95 to 1.09) [12].

It is now important to assess whether these benefits persist, and to provide adequate reassurance about longer term safety. The need for caution when interpreting data on short term outcome alone was highlighted recently by the long term follow up of babies at risk of chronic lung disease, or with established chronic lung disease, who were treated with early high-dose dexamethasone after birth [13]. Because trials quickly showed there were obvious short term benefits, including reductions in ventilator and oxygen dependence and in chronic lung disease, steroids were increasingly adopted world-wide, for more than fifteen years. When studies with long term follow up were eventually reported they showed no significant increase in the overall number of long term survivors, but some reported a clear increase in the number of survivors with cerebral palsy [14].

Magnesium sulphate is associated with cerebral vasodilatation, and is a blocker of N-methyl-D-aspartate (NMDA) receptors in the brain, the pathway for anoxic cell damage. Magnesium sulphate could therefore effect later neurodevelopment of children exposed in utero. Potential effects on the baby may also depend on gestational age at exposure to magnesium sulphate. Case control studies have suggested that maternal magnesium sulphate administra- tion reduces the risk of cerebral palsy for low birthweight (<1500 g) infants, [15-17] although a recent study suggests such exposure may actually increase cerebral palsy [18]. There is also concern about a possible increase in paediatric mortality following exposure to magnesium sulphate [19]. For low birthweight babies in utero exposure may therefore be associated with 'survival of the fittest', although this has been disputed [20]. As the aetiology of cerebral palsy is different for preterm and term babies, even if the reduction in cerebral palsy for the former is real, we cannot assume there will be similar benefits for term babies.

The hypothesis that magnesium sulphate reduces the risk of cerebral palsy for preterm babies is being tested in ongoing trials in which women at risk of very preterm birth are randomised to magnesium sulphate or placebo $[21,22]$. These studies will recruit relatively few women with pre-eclampsia, however, and will not provide evidence on the effects of exposure nearer to term. Data from the first of these studies are now available [23]. In this study 1062 women at risk of delivery before 30 weeks gestation were allocated either magnesium sulphate or placebo. There was a moderate, although non-significant, reduction in the risk of death or cerebral palsy (RR 0.83, $95 \%$ CI 0.66 to 1.03 ). There was, however, a significant reduction in the proportion of children with substantial gross motor dysfunction (RR $0.51,95 \%$ CI 0.29 to 0.91 ).

Magnesium sulphate is already widely used. In the light of data from the Magpie Trial [12] on outcome at discharge from hospital, its use is likely to increase in years to come. The Magpie Trial Follow Up Study aims to provide reliable information about the longer term outcome for women and children. As there are relatively few reliable data about the morbidity and mortality associated with preeclampsia, these data will also enhance our understanding of the long term implications of pre-eclampsia.

\section{Trial design Objectives}

The main objective is to assess whether in utero exposure to magnesium sulphate has a clinically important effect on the child's chance of surviving without major neurosensory disability. The specific hypotheses are whether exposure to magnesium sulphate affects:

- the risk of the child dying

- the risk of severe cerebral palsy

- the risk of blindness or deafness

- the risk of developmental delay 
For some children we also aim to collect information that will allow us to compare less severe, but nevertheless important, differences in developmental progress between the two treatment groups. In addition, we will assess whether magnesium sulphate has any longer term impact on outcome for the women.

Other objectives are to explore the long term consequences of pre-eclampsia, and risk factors for adverse outcome, for both the woman and her child. Also, to develop and assess affordable and appropriate strategies for follow up of large numbers of children recruited to perinatal trials, particularly in low and middle income countries. We will develop and evaluate strategies for using the Ages and Stages Questionnaires (ASQ) [24] in Latin America, Africa and Asia. We will also modify the ASQ [24] into a simple low cost tool for use in low income countries, and assess its performance.

\section{Study sample}

The Magpie Trial recruited 10,141 women at 175 centres in 33 countries. Five women were excluded from the initial analyses, 4 because no data were available and 1 woman was entered into the wrong trial. Of the remaining 10,136, 8799 women (87\%) were randomised before delivery, and 1337 (13\%) were randomised in the first 24 hours after delivery. Outcome at discharge from hospital was available for 10,110 women, 8775 (87\%) of whom were randomised before delivery. These 8775 women gave birth to 9153 babies, of whom 9024 (98.6\%) were included in the analysis of outcome up to discharge from hospital. Overall, 7883 of the children whose mothers were randomised before delivery were alive at discharge from hospital, and for another 2 our last information was that they were still in hospital.

The 1337 women randomised after delivery gave birth to 1418 babies, of whom 143 (10\%) were stillbirths or neonatal deaths.

For a variety of reasons, follow up is not possible at some centres. In others, due to the difficulty of tracing women, only those randomised in the last year of recruitment (December 2000-November 2001) will be contacted. Overall, we estimate 2800-3350 families will be eligible for assessment in the Follow up Study, regardless of whether recruitment was before or after delivery. A further 280-335 children would have been eligible for the follow up study if they had survived until discharge.

For the main objective, assessing the effects of in utero exposure to magnesium sulphate, the analyses will include only children whose mothers were randomised before delivery. The assessment of long term outcome for the woman will include data for all women, regardless of whether randomisation was before or after delivery. Assessment of the ASQ will also be based on data for all children.

\section{Sample size}

Overall, we estimate 2800-3350 families will be eligible for assessment in the Follow up Study. A further 280-335 children would have been eligible for the follow up study if they had survived until discharge, and the total sample size for the children is therefore $3080-3685$.

For children who were alive at discharge from hospital outside the UK, we anticipate $20 \%$ loss to follow up. For the 800 children in the UK we expect $<1 \%$ losses to follow up (for mortality). In the UK, although non-response to the ASQ may be $10 \%$ some information about serious disability will be available from general practitioners. Loss to follow up for disability in the UK is therefore likely to be $0-5 \%$. So, we expect to have data for 2400-2840 children who were alive at discharge from hospital, 2090-2475 of whom will have been born to women randomised before delivery. In total, this means we will have data on death or disability for 2680-3175 children, 2370-2810 of whom will have been born to women randomised before delivery.

Many of these children are high risk. For example, one third of births were $<34$ weeks. In the UK $94 \%$ of these very preterm babies survived until discharge from hospital, outside the UK $80 \%$ survived. We anticipate a further $3-5 \%$ of children may die after discharge, $15-25 \%$ of children may screen positive on the ASQ, [24,25] of whom $30-40 \%$ [24] may have neurosensory disability confirmed. This suggests that the composite primary outcome (death or neurosensory disability) may affect $20-25 \%$ of children in the placebo group. Power to detect a range of clinically important reductions in the treatment group are illustrated in table 1.

Even when adjusted for expected losses to follow up, the anticipated sample size of 3080-3685 children, of whom 2680-3210 will have been born to women randomised before delivery, will provide adequate power to detect a relative difference between the groups of at least $20 \%$ in the composite primary outcome.

How many screen negative children do we need to assess? We anticipate assessing 450-550 screen negative children and, at best, may be able to increase this to 700-800 children. Confidence intervals for a range of possible false negative rates in a sample of 500 screen negative children are shown in table 2 . 
Table I: Sample sizes for a range of power calculations for the composite primary outcome

\begin{tabular}{|c|c|c|c|c|}
\hline & \multicolumn{4}{|c|}{$25 \%$ reduction in risk of death or neurosensory disability } \\
\hline & $18 \% \rightarrow 13.5 \%$ & $20 \% \rightarrow 15 \%$ & $22 \% \rightarrow 16.5 \%$ & $24 \% \rightarrow 18 \%$ \\
\hline alpha 0.05, power $90 \%$ & 2840 & 2500 & 2230 & 2000 \\
\hline \multirow[t]{3}{*}{ alpha 0.05 , power $80 \%$} & 2140 & 1890 & 1684 & 1512 \\
\hline & \multicolumn{4}{|c|}{$20 \%$ reduction in risk of death or neurosensory disability } \\
\hline & $18 \% \rightarrow 14.4 \%$ & $20 \% \rightarrow 16 \%$ & $22 \% \rightarrow 17.6 \%$ & $24 \% \rightarrow 19.2 \%$ \\
\hline alpha 0.05 , power $80 \%$ & 3390 & 2990 & 2664 & 2390 \\
\hline \multirow[t]{3}{*}{ alpha 0.05, power $70 \%$} & 2376 & 2114 & 2114 & 1896 \\
\hline & \multicolumn{4}{|c|}{ I5\% reduction in risk of death or neurosensory disability } \\
\hline & $18 \% \rightarrow 15.3 \%$ & $20 \% \rightarrow 17 \%$ & $22 \% \rightarrow 18.7 \%$ & $24 \% \rightarrow 20.4 \%$ \\
\hline alpha 0.05 , power $60 \%$ & 3870 & 3410 & 3036 & 2722 \\
\hline
\end{tabular}

Table 2: Confidence intervals for a range of possible false negative rates in a sample of 500 screen negative children

\begin{tabular}{cc}
\hline $\begin{array}{c}\text { Observed false negative rate } \\
\text { (\% of cases among screen negatives) }\end{array}$ & $\begin{array}{c}\mathbf{N}=\mathbf{5 0 0} \\
\text { (95\% confidence interval) }\end{array}$ \\
\hline $5 \%$ & $3.4 \%$ to $7.3 \%$ \\
$2 \%$ & $1.1 \%$ to $3.6 \%$ \\
$1 \%$ & $0.4 \%$ to $2.3 \%$ \\
$0 \%$ & $0 \%$ to $0.8 \%$ \\
\hline
\end{tabular}

\section{Outcome measures}

The most extreme outcomes for the child are death, and then major neurosensory disability. The two are not independent, as children with major neurosensory disability have a high mortality and, particularly for children born too early, reduction in mortality may be at the cost of an increase in neurosensory disability. The primary outcome for this follow up study is the composite outcome of death or major neurosensory disability at 18 months.

Secondary outcomes for the baby are death alone, each of the individual measures of neurosensory disability alone, delayed speech, other important disability and contact with health services. Developmental attainment will be compared across the treatment groups for the subset of children for whom these data are available. Outcomes for the mother are death or serious morbidity potentially related to pre-eclampsia, other serious morbidity, hypertension requiring treatment, any reported hypertension, subsequent pregnancies and their outcome, and contact with health services.

\section{Definition of the primary outcome}

Our definition of death or major neurosensory disability needs to take account of several complex issues. These include the wide range of duration of follow up for each child, age at death, how severe the disability has to be, and what age does a child have to be before anyone can be confident that s/he does, or does not have, such disability. To ensure that our primary outcome is clear and unambiguous, we define below death and neurosensory disability.

Such a composite end point needs to be expressed at a single concordant age. The time point of 18 months (corrected for gestation at birth) was chosen because it strikes the best balance between feasibility of follow up and certainty in the diagnosis of neurosensory disability. Children who are assessed when they are older then 18 months will be included in the primary outcome. For example, if a child is assessed as having neurosensory disability when $\mathrm{s} /$ he is 24 months, or older, it is reasonable to assume that this would have been diagnosed at 18 months, had the child been seen then. Conversely, if a child seen at 24 months has no neurosensory disability, it is reasonable to assume the same would have been true at 18 months. However, if there was an obvious cause for the neurosensory disability, such as trauma or meningitis, that occurred when the child was older than 18 months, the child would be excluded from the analysis of the primary outcome (these children would be included in 'other disabilty', see below). Data for children assessed at 12-17 months of age will also be included in the primary outcome. If a child of 12 months is blind, deaf or unable to sit without support (developmental progress suggestive of cerebral palsy), s/he is likely to have the composite primary outcome by the age of 18 months. Surviving 
children assessed only when less than 12 months old will not be classified as to whether or not they have neurosensory disability, as they are too young for any such judgement to be reliable. Deaths when the child is older than 18 months will not be included in the analysis of primary outcome.

\section{Death}

Deaths from defects arising during conception or embryogenesis will be excluded from the main analysis, but will be included in a sensitivity analysis. Ideally only children who were alive in utero at the time the woman was randomised will be included. Unfortunately this was not always known for certain in the Magpie Trial. The analysis of deaths will therefore exclude babies who were likely to have died in utero before trial entry. These are those for whom the fetal heart beat was not heard at trial entry, and who were a macerated stillbirth within 24 hours after trial entry. This same exclusion was used in the main study report [12]. Children who died after trial entry but before discharge from hospital, who would have been selected for follow up had they survived, will be included in the sample.

\section{Neurosensory disability}

Currently, there is no internationally agreed consensus on what constitutes 'major disability', or how it should be assessed. Our definition of major neurosensory disability is therefore based on commonly agreed components, combined with a pragmatic view of what is likely to be feasible and sensible to measure. For this study we defined major neurosensory disability as the presence of one or more of severe cerebral palsy, blindness, deafness, and severe developmental delay. Disability due to defects arising during conception or embryogenesis will be excluded from the main analysis, but will be included in a sensitivity analysis.

We have included only severe cerebral palsy in our definition of major neurosensory disability. Although the diagnosis of cerebral palsy is problematic for younger children, our aim is to detect children with major functional abnormalities, which can be done at an early age [26]. Cerebral palsy will be diagnosed if the child has nonprogressive motor impairment characterised by abnormal muscle tone and a decreased range or control of movements. For example, most healthy children are sitting confidently, and unsupported, by 8 months (corrected for gestation) while those with severe cerebral palsy are not doing this by 12 months. Whenever possible, we will also classify children who were 12 months or older as likely to have mild, moderate or severe cerebral palsy. For example, at 2 years of age severe cerebral palsy will include nonambulant children who are never likely to walk, moderate cerebral palsy will include non-ambulant children who are expected ultimately to walk independently, and mild cerebral palsy will include ambulant children at 2 years of age. This will increase the clinical relevance of our results, and facilitate amalgamation of our data with those from other relevant trials $[21,22]$.

Studies have differed over whether to take a developmental quotient of either more than two standard deviations or more than three standard deviations below normal as an indication that a child has 'severe disability' [27]. We have defined severe developmental delay as being more than two standard deviations from the mean, which indicates progress is only what would be expected of a child two thirds that age. For example, this would assess a child who could not walk unaided at two as comparably disabled to a child who could not sit unaided by twelve months (corrected for gestation). This has the advantage of simplifying the amalgamation of the results from centres that assess children at different ages.

Blindness is defined as binocular visual acuity of less than $6 / 60$. Deafness is defined as a hearing loss of $50 \mathrm{~dB}$ or more in the better ear. In practise this is the threshold at which children normally benefit from a hearing aid (although there will, of course, be a few children so deaf that an aid offers little benefit).

Severe developmental delay will be defined on the revised Bayley Scales of Infant Development (BSID-II) [28] as a Mental Development Index (MDI) score <70 (>2 SD below mean of 100) [28]. On the Griffiths Scale, [29] severe developmental delay will be defined as a global developmental quotient (DQ) of $>2$ SD below the mean. Equivalent definitions will be applied to the Denver Developmental Screening Test-2 (DDST-II), [30] and its derivatives.

\section{Likely disability}

We anticipate data will be incomplete for some children, therefore there will also be a category of 'likely disability'. This will include: children for whom data are incomplete (such as children with significant developmental delay not subjected to formal qualitative developmental assessment), and those assessed at 12-17 months, for whom major disability seems likely as assessed by 2 independent experts. These children will be included in the primary outcome.

\section{Definitions of secondary outcomes for the child Other disability}

Our definition of 'other disability' will include two groups of children: those with non-neurosensory disability and those with mild-moderate neurosensory disability. 
It has been argued that indicators of non-neurosensory disability should be included within a broad inclusive definition of 'severe disability' [27]. Examples of non-neurosensory disability include the need for continuous supplemental oxygen, breathing support, renal dialysis, and tube or parenteral feeding. The provision of such treatments is variable and discretionary, however, so that even though their provision definitely indicates disability, it seems better not to include them in the definition adopted for the Magpie Trial. The same might, on pragmatic grounds, also be said about taking "the presence of seizures more than once a month despite appropriate treatment" as another objective marker of severe disability. In practice, most children with these problems will have already been classified as having a 'severe disability' on other grounds. We will report children who have these problems. Those who also meet our definition, above, of neurosensory disability, will be included in our primary outcome. Those who do not will be included in the secondary outcome of 'other disability'.

It is sometimes difficult to identify with certainty mild to moderate disability in children less than two years old. Study centres will therefore be encouraged to assess as many children as possible at 24 months, or more, after they were due to be born. In some collaborating centres this will not be feasible. So, when assessing whether a child has mild to moderate disability all information on children assessed 18 months, or more, after they were due to be born will be used. Also, as most children will only be assessed in more detail if they 'fail' the ASQ, there may be some under ascertainment of this more moderate disability. There is, however, no reason why ascertainment should differ in the two treatment groups.

Our definition of other disability will include any child, aged 18 months or more:

- with non-severe cerebral palsy

- with reduced mobility - even if not identified as having cerebral palsy

- having more than 1 seizure a month despite treatment

- unable to feed themselves for any reason after 2 years of age

- having a Bayley Psychomotor Developmental Index (PDI) score >2 SD below normal

- with other disability not included in neurosensory disabilty (see above)

\section{Delayed speech}

Isolated speech delay is not uncommon. When language comprehension is good, however, there is seldom a lasting problem. We will therefore report how many children in each treatment group had a vocabulary of less than 10 words at two years, or more, after they were due to be born, but have no other reason for being classified as having a disability. This analysis will be restricted to children assessed when they were at least 24 months old, as language development for younger children is very variable.

\section{Contact with health services}

The number and reasons for clinic attendance and hospital admission will be compared between treatment groups.

\section{Definitions of secondary outcomes for the mother Death of the women}

This will include all deaths after discharge from hospital and those before discharge from hospital of women who would have been selected for follow up, had they survived.

Serious morbidity for the women, potentially related to pre-eclampsia Serious maternal morbidity will include women with eclampsia or other serious morbidity potentially related to pre-eclampsia before discharge from hospital, as well as those with serious morbidity after discharge. Serious morbidity potentially related to pre-eclampsia before discharge was pre-defined as one or more of respiratory depression, respiratory arrest, pneumonia, cardiac arrest, coagulopathy, renal failure, liver failure, pulmonary oedema and cerebral haemorrhage. Serious morbidity after discharge will include renal problems, stroke, and severe hypertension.

\section{Other serious morbidity}

Other serious morbidity will include women with thromboembolic disease, major psychiatric problems, major infection, and cancer.

\section{Hypertension for the women}

Hypertension will be defined as any raised blood pressure since discharge sufficient to require antihypertensive treatment. Those women taking antihypertensive treatment at the time of assessment will also be reported.

\section{Reproductive morbidity}

Reproductive morbidity will include miscarriage, ectopic pregnancy, and gynaecological problems.

\section{Contact with health services}

The number and reasons for clinic attendance and hospital admission will be compared between treatment groups. 


\section{Assessments How the children will be assessed}

The main aim of the follow up study is to investigate any effect of magnesium sulphate on death or major neurosensory disability in the surviving children. In many countries the only way to check whether the child is still alive will be to contact the family. As well as being a means of finding out whether the child is alive, this makes it possible to find out how the child is progressing, and will also provide information about the health status of the mother. All assessments of women and children will be made blind to treatment group.

Children will be screened for serious disability using the ASQ, [24] or a shortened version of the ASQ (see below). In the UK and other developed countries the questionnaires will be sent to the family by post. Elsewhere, the questionnaires will be administered by telephone, in a clinic, or during a home visit. If the family has moved and cannot be contacted, information will be collected, whenever possible, from neighbours. If the woman is invited to attend a clinic this will be to a dedicated clinic to reduce waiting time. These clinics may provide a package of appropriate health care, such as family planning or immunisation, to encourage attendance. The family's travelling expenses will be reimbursed.

\section{When the children will be assessed}

Neurosensory disability (a serious problem with vision, hearing or movement control) can be recognised with some confidence within 18 months of birth, especially if any preterm baby is only examined 18 months after the expected date of birth. We will therefore encourage our collaborators to assess as many children as possible when they are 18 months, or more. The age at which first contact is made with the family will be determined locally, and will primarily depend on what is feasible. It is not possible to contact and assess the children at a single age across the study. We will therefore take the pragmatic approach that any information about outcome after discharge from hospital is better than none. For children being assessed when they are less than or equal to 24 months and who were born before 37 completed weeks, an adjustment will be made for gestation at birth.

\section{When the mothers will be assessed}

To contact the child it is usually necessary to contact the mother. The women will be asked a few simple questions about their own health, at the same time as any assessment of their child. Whether mothers of children who died are contacted will be at the discretion of the local collaborators in consultation with their ethics committee.

\section{How outcome will be assessed}

Screening with the ages and stages questionnaires

Children will be screened using the Ages and Stages Questionnaires (ASQ) [24]. The ASQ [24] is a series of 19 questionnaires designed to be completed by parents or primary caregivers, but they perform equally well if completed with assistance from a researcher or a health care worker. The questionnaires are validated for intervals from 4 months-5 years, adjusted for gestation at birth up to 24 months. Each is valid for eight weeks, four weeks either side of the target age.

Each of the 19 age-targeted questionnaires contains 30 developmental questions covering five domains: communication, gross motor, fine motor, problem solving and personal-social. An additional section has 8 questions addressing general parental concerns. The questionnaires are written in simple, straightforward language and illustrations assist parents in understanding some items. For each developmental item the response requested is 'yes', 'sometimes' or 'not yet'. To score the responses, each is converted to a point value, which is totalled within the domain, and the totals are compared to established screening cut-off points. If one or two questions in a domain have not been answered, a ratio score can be computed. If more than two items are omitted, the domain cannot be scored. If one or more domains could not be scored, the child will be treated as screen positive, and invited to have a full assessment. If the ASQ is completed outside the 8 week window for which it is valid (see above), this will also be treated as 'screen positive'. The 18 month questionnaire is appendix 1 [Additional file: 1 ].

The ASQ can accurately identify children who may have developmental disorders, and therefore need further evaluation. Reported sensitivity for mild to moderate developmental delay ranges from 38-90\%, and specificity ranges from $81-91 \%$ [24]. Positive predictive value ranges from $32-64 \%$ [24]. Factors in this variation between questionnaires include random errors, and how well the test performs at each age. For questionnaires at 12-24 months, sensitivity is reported to range from $65 \%$ to $85 \%$ and specificity from $81 \%$ to $90 \%$. Our expectation is that the ASQ will have a high sensitivity and negative predictive value in our population. For example, a child who is completely blind, or completely deaf, or has severe cognitive delay or severe cerebral palsy will fail at least one domain within the ASQ, and so will be screen positive. Overall sensitivity to identify delayed development is $72 \%$, but when used to identify children with established developmental delay sensitivity has been shown to be higher, at 96\%, [24] and for ex-preterm children it is $90-100 \%$ [25].

The ASQ has major advantages for use in large international trials. It is flexible, being validated for use across a 
range of ages and cultures, although primarily in North America, and for both preterm [25] and term children [24]. It is available in English, Spanish and French. Performance is not reduced if the interviewer paraphrases the exact wording, or if culturally inappropriate questions are altered or dropped [24]. It is also low cost. The age at which children are assessed can be tailored to the needs of individual centres, so no child should be lost to follow up merely because it was not possible to contact the family within a narrow time window.

\section{Shortened ages and stages questionnaires}

For centres where use of the full ASQ is not feasible, we have developed shorter ASQs for 8, 10, 12, 14, 16, and 18 months. To facilitate administration, these are provided to collaborators in a booklet (see 1). Additional short ASQs are available up to 36 months. The shortened ASQ uses three questions in each of three domains: gross motor, communication and problem solving, with 5 overall questions. To pass, the child must achieve at least one 'yes' in each domain. The use of 38 questions in the full ASQ is designed primarily to identify moderate or subtle delay in development. Reducing the number of questions may reduce performance for these more subtle effects, but this small number of questions should still be adequate for detecting gross abnormalities [31].

Children who screen positive on the ages and stages questionnaires As severe disability has a low prevalence, positive predictive values are likely to be at the low end of the reported range. We cannot therefore assume that all screen positive children are indeed severely disabled. The screen positive children will thus be invited for a clinical and neurodevelopmental assessment using the Bayley Scales of Infant Development [28] or the Griffiths Tests [29]. If neither of these can be used, an alternative test may be used. If no test is feasible, we will rely on clinical history and examination alone. Families will not be told their child has 'failed' the screen, as this would cause unnecessary concern. They will be invited to have the assessment in a way that is as convenient as possible for them.

Children who screen negative on the ages and stages questionnaires Our aim is to identify children with severe disability. It is reasonable to assume that the ASQ will have a higher negative predictive value when used to identify severe disability, than when used to identify mild developmental delay. We will test this assumption by assessing a sample of screen negative children. In the UK, sample selection will be based on geographic location at trial entry. Outside the UK, some centres will assess all screen negative children. Others will assess a predefined sample.

\section{Dealing with the family when the child has severe disability}

The ASQ is a screening tool, and many of the children who fail the questionnaire (screen positive) will be normal. Due to this risk of being false positive, parents and carers will not be told the child has a problem based on the ASQ alone. Many of the children with disability in the study will already have been identified by the health services, and the family or carer will be aware of the child's problems and diagnosis. In this situation, the family should be dealt with sensitively. The child should not be required to have any unnecessary testing, and any questions or concerns should be responded to.

The study may identify some children with severe disability who, despite their considerable problems, have not yet been detected by the health services. It is likely that the family or carer of such a child will be aware of the child's difficulties. The diagnosis of severe disability will usually be made by the paediatrician doing the assessment. S/he should then explain it to the family, as would happen in normal clinical practice. If it is appropriate to refer the child to additional health services, and the family agree, this should again be done in accordance with normal clinical practice. If necessary, the Magpie Trial Co-ordinating Centre should be contacted for advice.

\section{Ethics approval and consent}

The information leaflet given to women before they gave consent to recruitment to the Magpie Trial stated that women might be contacted later to find out how they and their child are. In some countries ethics committees have agreed women need not be asked again for consent to being followed up. All women and carers have the option to opt out of the study, if they wish. Some centres prefer to ask the women to consent again to the follow up study. This decision should be made in consultation with the relevant local ethics committee.

\section{Data management}

Data will be processed and cleaned as they accumulate. Queries will be discussed with the local co-ordinator whenever possible. There will be no interim analyses.

A paediatrician will review data for each child who died, or is thought to have neurosensory disability or other disability. If there is any uncertainty, a second independent expert will review the data, and the outcome will be determined by consensus. To check for consistency, the second expert will also review data for a random $10 \%$ sample of these children. An obstetrician will review data for women who died or had serious morbidity. If there is any uncertainty, a second independent expert will review the data, and the outcome will be determined by consensus. 


\section{Planned analyses}

Completeness of follow up at each participating centre will be described. Centres that return data for either $>20 \%$ of all families selected for follow up, or $>20 \%$ of families where the mother was randomised before birth, will be included in the main analyses. Characteristics at trial entry and outcome at discharge from hospital will be compared for women and children who were successfully contacted, those lost to follow up, and the trial overall. How and when each family was contacted, and what information is available for each person will be presented.

The main analyses of primary and secondary measures of outcome will be based on the groups as randomly allocated regardless of the amount of magnesium received (an intention to treat comparison).

The analysis of children's death alone will include all deaths and will take account of the age at death, using logrank survival analysis. This will allow for the fact that different children were of variable age when last known to be alive. Cause of death will be classified [32,33] and, where possible, presented, as will information about disability prior to death.

The groups will be compared for each type of neurosensory disability, as defined above, and for children with multiple disabilities. Mortality and disability by gestation at birth will be presented.

For the primary outcome statistical significance will be taken as the 5\% level, and for secondary outcomes the $1 \%$ level. The main analyses will be by outcome for each pregnancy. For multiple births outcome will be taken as the worst for any of the children.

We will compare data from those children who were subject to formal neurodevelopmental testing. As such information will be available for screen positive children, but for only a sample of screen negative children, these scores will have an artificially truncated distribution. The median and distribution of scores will be compared between the treatment groups, for each age of assessment. Whenever possible, numerical information on developmental progress will be converted to a z-score using local control data. If there are no local data, we will use the overall mean and SD to compute a $\mathrm{z}$-score.

We expect to have detailed information on at least 2500 children assessed using the full ASQ questionnaire. The aim is to compare the progress being made by children in the two treatment groups using a rank scoring approach. This will not assume that the spread of development is normally distributed. To allow for the fact that children will be tested at different ages, each will be 'scored' by how many points s/he scored above or below the cut off for that domain for a child of that age (after adjustment for gestation at birth if $<24$ months). Children for whom we have only a short ASQ will be excluded from this analysis, as will those whose assessment was outside the appropriate time window.

The possible influence of dose of magnesium sulphate to which the child was exposed between trial entry and delivery will be explored, recognising that this dose may be influenced by the developing clinical situation. The full plan for this analysis will be formulated before the data are unblinded.

For the women, deaths will be presented as a graph of cumulative mortality. Causes of death will be described.

\section{Sensitivity and subgroup analyses}

Sensitivity analyses for the composite primary outcome of death or neurosensory disability for the child will be:

- excluding children classified as having 'likely disability'

- including children whose death or neurosensory disability originated during conception or embryogenesis

- excluding data from centres where $<90 \%$ of randomised children were selected for follow up, and selection for follow up was not based on a time frame

- including data from centres excluded from the main analyses because a high proportion $(\geq 80 \%)$ could not be contacted

- considering each child of multiple births rather than just the one with the worst outcome

Subgroup analyses for the composite primary outcome of death or neurosensory disability for the child will be by:

- severity of the mother's pre-eclampsia at trial entry (severe, moderate, mild)

- whether magnesium sulphate had been given in the 48 hours before trial entry

- gestation at birth (<34 weeks, $\geq 34$ weeks)

- perinatal mortality in the country (low, medium or high)

- whether the mother received trial treatment by the intramuscular or the intravenous route

- multiple or singleton births 
- degree of difficulty in data collection (possible at the first attempt, reminders, or indirect contact only).

Severity of pre-eclampsia is defined in fig [1]. Severe preeclampsia is as defined in the original trial protocol. The definition of moderate and mild pre-eclampsia was agreed (although not published) before the final analysis of outcome at discharge from hospital. These two groups were previously reported as 'non-severe' pre-eclampsia [12]. Perinatal mortality for each country will be as reported by WHO, [34] as it was in the analysis of outcome at discharge from hospital [12]. Low perinatal mortality will be taken $<20$ deaths/ 1000 births, moderate as $20-40 / 1000$ births and high as $>40 / 1000$ births.

For the women, subgroup analyses for the main outcome of death or serious morbidity will be by:

- severity of pre-eclampsia at trial entry (see Box 1)

- gestation at trial entry (<34 weeks, $\geq 34$ weeks)

- perinatal mortality in the country (low, medium or high)

Additional analyses will evaluate performance of the full ASQ and the shortened ASQ.

\section{Trial management}

\section{Magpie Trial follow up study management group}

This group meets every 2-3 months to assist with day to day running of the trial, and to prepare reports for the steering committee. Membership: Douglas Altman ${ }^{1}$, Nina Armstrong2, Mike Clarke ${ }^{3}$, Lelia Duley ${ }^{2}$, Barbara Farrell (Chair) ${ }^{2}$, Alastair Gray ${ }^{4}$ Edmund Hey ${ }^{5}$, James Neilson ${ }^{6}$, Judit Simon ${ }^{4}$, Rebecca Smyth2 ${ }^{2}$ Patsy Spark ${ }^{2}$ and Ly-Mee $\mathrm{Yu}^{1}$.

${ }^{1}$ Centre for Statistics in Medicine, Oxford; ${ }^{2}$ Magpie Trial Co-ordinating Centre; ${ }^{3} \mathrm{UK}$ Cochrane Centre, Oxford; ${ }^{4}$ Centre for Health Economics Research, Oxford; ${ }^{5}$ paediatrician, Newcastle; ${ }^{6}$ Department of Obstetrics and Gynaecology, Liverpool.

\section{Magpie Trial follow up study steering committee}

The overall progress of the follow up study will be monitored by a scientific and administrative steering committee. Membership: Douglas Altman, Rory Collins, Lelia Duley, Richard Lilford, Jack Moodley, James Neilson, Ian Roberts, Stephen Robson, Peter Rubin (chair), James Thornton, Sara Twaddle, and Isabel Walker. Observers are Anna Karaoglou, Barbara Farrell, a representative from the Department for International Development and José Villar. The UK Medical Research Council convenes this committee.

\section{Statisticians}

Douglas Altman, Ly-Mee Yu. Centre for Statistics in Medicine, Oxford.

\section{Publication plan}

The success of the Magpie Trial Follow Up Study depends upon the active collaboration of a large number of people, particularly the co-ordinators and other staff at each participating centre. For this reason, authorship of presentations and reports related to the study will be in the name of the collaborative group. The final Follow Up Study results paper will name local co-ordinators as well as those involved in central co-ordination and trial management. Co-ordinators who provided data will be named, even if all woman and children for whom they collected data were excluded from the analyses. Certificates of collaboration will be provided to those who have made a substantial contribution but whose name is not on the final report.

Papers on other aspects of the follow up study will be published with those who made substantive contributions being named as authors. These papers will make appropriate acknowledgement of the contribution of the collaborative group.

\section{Discussion}

The Magpie Trial Follow Up Study was launched in March 2002, and now involves collaborators in 19 countries. Data collection will close at the end of 2003, and results are expected to be available by the end of 2004 .

\section{Funding}

The Follow Up Study is funded by the MRC and the Department for International Development, with support from WHO.

Appendix 118 month long child's questionnaire [see Additional file: 1]

Appendix 2 Child's booklet of shortened questionnaires for ages 8-18 months [see Additional file: 2

Appendix 3 Paediatric assessment form [see Additional file: 3$]$

Appendix 4 Woman's questionnaire [see Additional file: 4]

\section{Competing interests}

None declared. 


\section{Additional material}

\section{Additional file 1}

Click here for file

[http://www.biomedcentral.com/content/supplementary/14712393-4-5-S1.pdf]

\section{Additional file 2}

Click here for file [http://www.biomedcentral.com/content/supplementary/14712393-4-5-S2.pdf]

\section{Additional file 3}

Click here for file

[http://www.biomedcentral.com/content/supplementary/14712393-4-5-S3.pdf]

\section{Additional file 4}

Click here for file

[http://www.biomedcentral.com/content/supplementary/14712393-4-5-S4.pdf]

\section{References}

I. World Health Organization International Collaborative Study of Hypertensive Disorders of Pregnancy: Geographic variation in the incidence of hypertension in pregnancy. Am J Obstet Gynecol 1988, I 58:80-83.

2. The National Institute for Clinical Excellence, The Scottish Executive Health Department, The Department of Health Social Services and Public Safety Northern Ireland: Why mothers die 1997-1999: the fifth report of the Confidential Enquiries into Maternal Deaths in the United Kingdom London: RCOG Press; 2001.

3. Department of Health, Welsh Office, Scottish Office Department of Health, Department of Health and Social Services NI: Why mothers die: Report on Confidential Enquiries into Maternal Deaths in the United Kingdom, 1994-1996. London: TSO 1998.

4. Duley L: Maternal mortality associated with hypertensive disorders of pregnancy in Africa, Asia, Latin America and the Caribbean. Br J Obstet Gynaecol 1992, 99:547-53.

5. Department of Health: Confidential Enquiry into stillbirths and deaths in infancy. London: Department of Health 1996.

6. Crowther CA: Eclampsia at Harare Maternity Hospital. An epidemiological study. S Afr Med J 1985, 68:927-929.

7. Douglas KA, Redman CW: Eclampsia in the United Kingdom. BM] 1994, 309:1395-1400.

8. Duley L, Gulmezoglu AM: Magnesium sulphate versus lytic cocktail for eclampsia (Cochrane Review). The Cochrane Library 2003:CD002960.

9. Duley L, Henderson-Smart D: Magnesium sulphate versus phenytoin for eclampsia (Cochrane Review). The Cochrane Library 2003:CD000I28.

10. Duley L, Henderson-Smart D: Magnesium sulphate versus diazepam for eclampsia (Cochrane Review). The Cochrane Library 2003:CD000I27.

II. Duley L, Gulmezoglu AM, Henderson-Smart DJ: Magnesium sulphate and other anticonvulsants for women with pre- eclampsia (Cochrane Review). The Cochrane Library 2003:CD000025

12. Magpie Trial Collaborative Group: Do women with pre-eclampsia, and their babies, benefit from magnesium sulphate? The Magpie Trial: a randomised placebo-controlled trial. Lancet 2002, 359: $1877-1890$.

13. Halliday HL, Ehrenkranz RA: Early postnatal (<96 hours) corticosteroids for preventing chronic lung disease in preterm infants (Cochrane Review). The Cochrane Library 2003:CD00I I 46.

14. American Academy of Pediatrics. Committee on Fetus and the Newborn: Postnatal corticosteroids to treat or prevent chronic lung disease in preterm infants. Pediatrics 2002, 109:330-338.

15. Nelson KB: Magnesium sulfate and risk of cerebral palsy in very low-birth-weight infants. JAMA 1996, 276: 1843-1844.

16. Nelson KB, Grether JK: Can magnesium sulfate reduce the risk of cerebral palsy in very low birthweight infants? Pediatrics 1995, 95:263-269.

17. Schendel DE, Berg CJ, Yeargin-Allsopp M, Boyle CA, Decoufle P: Prenatal magnesium sulfate exposure and the risk for cerebral palsy or mental retardation among very low-birth-weight children aged 3 to 5 years. JAMA 1996, 276:1805-1810.

18. Lemons JA, Vohr BR, Stevenson DR, Poole K, Das A, Bauar CR, et al.: In utero magnesium exposure: effects on ELBW infants at 18 months adjusted age. Pediatr Res 200I, 49::388.

19. Mittendorf R, Covert R, Boman J, Khoshnood B, Lee KS, Siegler M: Is tocolytic magnesium sulphate associated with increased total paediatric mortality? Lancet 1997, 350:1517-1518.

20. Grether J, Hoogstrate J, Selvin S, Nelson KB: Magnesium sulphate tocolysis and risk of neonatal death. Am J Obstet Gynecol 1998 , 178: I-6.

21. Benichou J, Zupan V, Fernandez H, Marpeau L, Marret S: Tocolytic magnesium sulphate and paediatric mortality. Lancet 1998 , $351: 290-291$.

22. Crowther C, Hiller J, Doyle L, Lumley J, Carlin J: Tocolytic magnesium sulphate and paediatric mortality. Lancet 1998, 35 I:29I.

23. Crowther C, Hiller J, Doyle L, Haslam R, for the Australian Collaborative Trial of Magnesium Sulphate (ACTOMgSO4) Collaborative Group: Effect of magnesium sulfate given for neuroprotection before preterm birth. A randomized controlled trial. JAMA 2003, 290:2669-2676.

24. Squires J, Potter L, Bricker D: The ASQ user's guide for the Ages and Stages Questionnaires: a parent-completed, child monitoring system 2nd edition. Paul Baltimore: Brookes Publishing Co; 1999.

25. Skellern CY, Rogers Y, O'Callaghan MJ: A parent-completed developmental questionnaire: follow up of ex- premature infants. J Paediatr Child Health 200 I, 37:125-129.

26. Stanley F, Alberman E, Eds: The epidemiology of the cerebral palsies. London: Heineman 1984.

27. A report of two working groups convened by the National Perinatal Epidemiology Unit and the former Oxford Regional Health Authority: Disability and perinatal care: measurement of health status at two years. Oxford: National Perinatal Epidemiology Unit 1994.

28. Bayley N: Manual for the Bayley scales of infant development. New York: Psychological Corporation 21993.

29. Griffiths R: The abilities of babies: a study in mental measurement. Revised reprint 1986 edition. Association for Research in Infant and Child Development 1986.

30. Frankenburg W, Dodds J, Archer P, Shapiro H, Bresnick B: The Denver II: a major revision and restandardization of the Denver Developmental Screening Test. Pediatrics 1992, 89:91-97.

31. Fooks J: Four key questions that identify severe disability. Arch Dis Child 1999, 80:67-68.

32. Alberman E, Blatchley N, Botting B, Schuman J, Dunn A: Medical causes on stillbirth certificates in England and Wales: distribution and results of hierarchical classifications tested by the Office for National Statistics. Br J Obstet Gynaecol 1997, 104: 1043-1049.

33. Alberman E, Botting B, Blatchley N, Twidell A: A new hierarchical classification of cases of infant deaths in England and Wales. Arch Dis Child 1994, 70:403-409.

34. WHO: Perinatal mortality: a listing of available information. WHO/FRH/ MSM/96.7 Geneva: WHO 1996. 


\section{Pre-publication history}

The pre-publication history for this paper can be accessed here:

http://www.biomedcentral.com/1471-2393/4/5/prepub

Publish with Bio Med Central and every scientist can read your work free of charge

"BioMed Central will be the most significant development for disseminating the results of biomedical research in our lifetime. " Sir Paul Nurse, Cancer Research UK

Your research papers will be:

- available free of charge to the entire biomedical community

- peer reviewed and published immediately upon acceptance

- cited in PubMed and archived on PubMed Central

- yours - you keep the copyright
BioMedcentral 